\title{
ON INTEGRAL INEQUALITIES ASSOCIATED WITH A LINEAR OPERATOR EQUATION
}

\author{
M. B. SUBRAHMANYAM
}

\begin{abstract}
In this paper we apply control theoretic concepts to formulate and solve a generalized problem for the determination of best possible constants in integral inequalities. We investigate the problem of existence of functions for which the best constant is attained, and also the conditions satisfied by these functions.
\end{abstract}

I. Introduction. In this paper we apply control theoretic ideas to a linear operator equation of the form $w=D u$ to determine the best possible constants in integral inequalities involving $w$ and $u$. The generalized approach of this paper encompasses all the previous cases considered [1-4]. On the other hand, it gives rise to integral inequalities in a number of new settings.

Consider the continuous linear operator

$$
D: L_{p}^{m}(A, \mu) \rightarrow L_{r}^{n}(B, \nu), \quad p, r>1,
$$

where $A \subseteq R^{k}$ and $B \subseteq R^{l}$ are subsets. $L_{p}^{m}(A, \mu)$ denotes the Banach space of $m$-dimensional measurable functions on $A$ with the norm

$$
\|u\|_{p}=\left[\int_{A}|u(t)|^{p} d \mu(t)\right]^{1 / p},
$$

where || denotes the finite-dimensional Euclidean norm, and $\mu$ a positive measure on $A$. Similar remarks apply to $L_{r}^{n}(B, \nu)$.

Let

$$
w=D u .
$$

Our problem is to select $u$ such that

$$
F(w, u)=\frac{\int_{A} \phi_{1}(u, t) d \mu(t)}{\left[\int_{B} \phi_{2}(w, x) d \nu(x)\right]^{\alpha}}
$$

is minimized, where $\alpha>0$, and $\phi_{1}, \phi_{2}$ are real-valued functions. Note that if the minimum value of (1.4) is $\beta>0$, we have the natural inequality

$$
\int_{A} \phi_{1}(u, t) d \mu(t) \geq \beta\left[\int_{B} \phi_{2}(w, x) d \nu(x)\right]^{\alpha},
$$

where $\beta$ is the best possible constant.

Received by the editors January 27, 1983.

1980 Mathematics Subject Classification. Primary 49A29; Secondary 26D15.

(C) 1984 American Mathematical Society $0002-9939 / 84 \$ 1.00+\$ .25$ per page 
II. Existence of best possible constant. We make the following assumptions:

(i) $\phi_{i}$ is continuous in its arguments for $i=1,2$. Also $\phi_{1}$ is convex in $u$ for each $t$.

(ii) Admissible $u$ are such that $\left|\int_{A} \phi_{1}(u, t) d \mu(t)\right|<\infty$.

(iii) $\phi_{2}(w, x) \geq 0$ for any $w$ which satisfies (1.3) for some admissible $u$.

(iv) $\nabla_{u} \phi_{1} \in L_{q}^{m}(A, \mu)$ for all $u \in L_{p}^{m}(A, \mu), 1 / p+1 / q=1$.

(v) For each $K<\infty$ there exists a $\nu$-integrable $g_{K}$ on $B$ such that for each $\|u\|_{p} \leq K$

$$
\left|\phi_{2}(w, x)\right| \leq g_{K} \quad \text { a.e. }[\nu] \text { on } B \text {. }
$$

(vi) $D: L_{p}^{m}(A, \mu) \rightarrow L_{r}^{n}(B, \nu)$ maps weakly convergent sequences into strongly convergent ones.

(vii) There exists $k>0$ such that

$$
\phi_{1}(c u, t)=c^{k} \phi_{1}(u, t), \quad \phi_{2}(c w, x)=c^{k / \alpha} \phi_{2}(w, x) .
$$

(viii) There is an admissible $u$ such that $0<\int_{B} \phi_{2}(w, x) d \nu(x)<\infty$.

THEOREM 1.1. Consider (1.3) and (1.4) along with assumptions (i)-(viii). Then there exists an admissible $u$ that minimizes (1.4).

PROOF. By the assumptions it is sufficient to exhibit a minimizing $u$ among all $u$ for which $\int_{B} \phi_{2}(w, x) d \nu(x)=M>0$. Let inf $\int_{A} \phi_{1}(u, t) d \mu(t)=J$ subject to $\int_{B} \phi_{2}(w, x) d \nu(x)=M$. Let $\left\{u_{n}\right\}$ be such that $\lim _{n \rightarrow \infty} \int_{A} \phi_{1}\left(u_{n}, t\right) d \mu(t)=J$ with $\int_{B} \phi_{2}\left(w_{n}, x\right) d \nu(x)=M$ for all $n$. Then a subsequence, still denoted by $\left\{u_{n}\right\}$, converges to some $u_{0} \in L_{p}^{m}(A, \mu)$ weakly. By assumption (vi), $w_{n}$ converges to $w_{0}$, and by assumption (v) and the Lebesgue dominated convergence theorem, we have $\int_{B} \phi_{2}(w, x) d \nu(x)=M$.

By assumption (i),

$$
\phi_{1}\left(u_{n}(t), t\right)-\phi_{1}\left(u_{0}(t), t\right) \geq \nabla_{u} \phi_{1}\left(u_{0}(t), t\right)\left(u_{n}(t)-u_{0}(t)\right)
$$

for almost all $t \in A$. Integrating both sides on $A$ and letting $n \rightarrow \infty$, we get, by assumptions (iv) and (v),

$$
\int_{A} \phi_{1}\left(u_{0}, t\right) d \mu(t) \leq \liminf _{n \rightarrow \infty} \int_{A} \phi_{1}\left(u_{n}, t\right) d \mu(t)=J .
$$

Corollary 1.1. Let $A=B, \mu=\nu$, and

$$
F(w, u)=\frac{\int_{A} \phi_{1}(w, u, t) d \mu(t)}{\left[\int_{A} \phi_{2}(w, t) d \mu(t)\right]^{\alpha}},
$$

where $\phi_{1}$ is convex in both $w$ and $u$ for each $t$ and $\nabla_{w} \phi_{1} \in L_{s}^{n}(A, \mu), 1 / r+1 / s=1$. Also assume that $\phi_{1}(c w, c u, t)=c^{k} \phi_{1}(w, u, t)$. Then, under the same assumptions of Theorem 1.1, there exists an admissible control $u_{0}$ minimizing $F(w, u)$.

III. Characterization of $u_{0}$. In this section we derive an equation that $u_{0}$ needs to satisfy. This is useful in synthesizing $u_{0}$ in particular cases. We consider equations (1.3) and (1.4) under the following assumptions.

(i) Admissible $u$ are such that $\left|\int_{A} \phi_{1}(u, t) d \mu(t)\right|<\infty$. 
(ii) $D: L_{p}^{m}(A, \mu) \rightarrow L_{r}^{n}(B, \nu)$ possesses an adjoint $D^{*}: L_{s}^{n}(B, \nu) \rightarrow L_{q}^{m}(A, \mu)$ $(1 / p+1 / q=1,1 / r+1 / s=1)$ such that for all $f_{1} \in L_{s}^{n}(B, \nu)$ and $f_{2} \in L_{p}^{m}(A, \mu)$, we have

$$
\int_{B}\left(f_{1}, D f_{2}\right) d \nu=\int_{A}\left(D^{*} f_{1}, f_{2}\right) d \mu .
$$

Here (, ) denotes the standard inner product.

(iii) $\left(w_{0}, u_{0}\right)$ minimizes $(1.4) \Rightarrow 0<\int_{A} \phi_{1}(u, t) d \mu(t)<\infty, 0<\int_{B} \phi_{2}(w, x) d \nu(x)$ $<\infty$.

(iv) Let $\phi_{1}$ be continuously differentiable in $u$ and measurable in $t$, and $\phi_{2}$ be continuously differentiable in $w$ and measurable in $x$.

(v) $\nabla_{u} \phi_{1} \in L_{q}^{m}(A, \mu)$ for all $u \in L_{p}^{m}(A, \mu), 1 / p+1 / q=1$ and $\nabla_{w} \phi_{2} \in L_{s}^{n}(B, \nu)$ whenever $w \in L_{r}^{n}(B, \nu), 1 / r+1 / s=1$.

(vi) There exist $\delta, k_{1}, k_{2}>0$ depending on $(w, u)$ such that for all $\|h\|_{r} \leq \delta$ and $\|\bar{u}\|_{p} \leq \delta$, we have

$$
\begin{aligned}
& \left\|\nabla_{u} \phi_{1}(u+\bar{u}, t)-\nabla_{u} \phi_{1}(u, t)\right\|_{q} \leq k_{1}\|\bar{u}\|_{p}, \\
& \left\|\nabla_{w} \phi_{2}(w+h, x)-\nabla_{w} \phi_{2}(w, x)\right\|_{s} \leq k_{2}\|h\|_{r} .
\end{aligned}
$$

THEOREM 3.1. Consider (1.3) and (1.4) along with the above assumptions. If $\left(w_{0}, u_{0}\right) \in L_{r}^{n}(B, \nu) \times L_{p}^{m}(A, \mu)$ minimizes (1.4), then

$$
\nabla_{u} \phi_{1}\left(u_{0}, t\right)-\alpha \lambda D^{*}\left(\nabla_{w} \phi_{2}\left(w_{0}, x\right)\right)=0 \text { a.e. }[\mu] \text { on } A \text {, }
$$

where

$$
\lambda=\frac{\int_{A} \phi_{1}\left(u_{0}, t\right) d \mu(t)}{\int_{B} \phi_{w}\left(w_{0}, x\right) d \nu(x)} .
$$

ProOF. Let $E=L_{r}^{n}(B, \nu) \times L_{p}^{m}(A, \mu)$. We have an equality constraint in $E$ given by (1.1). Associated with this is the cone $K_{1}$ of tangent directions. Associated with the functional (1.4) is the cone $K_{0}$ of directions of decrease. For the definitions of various terms, see [5]. If $H$ is a cone in $E$, then the dual cone $H^{*}=\{g \in$ $E^{*} \mid g(\tilde{p}) \geq 0$ for all $\left.\tilde{p} \in H\right\}$. The Dubovitskii-Milyutin theorem states that there exist $g_{0} \in K_{0}^{*}$ and $g_{1} \in K_{1}^{*}$, not both zero, such that for all $(w, u) \in E, g_{0}(w, u)+$ $g_{1}(w, u)=0$.

A. Cone of directions of decrease. By assumption (iii) we can minimize the alternative cost functional

$$
G(w, u)=\ln \int_{A} \phi_{1}(u, t) d \mu(t)-\alpha \ln \int_{B} \phi_{2}(w, x) d \nu(x) .
$$

By assumptions (iv)-(vi), the Fréchet derivative of $G$ at $\left(w_{0}, u_{0}\right)$ is given by

$$
\begin{aligned}
G^{\prime}\left(w_{0} u_{0}\right)(w, u)= & \frac{\int_{A}\left(\nabla_{u} \phi_{1}\left(u_{0}, t\right), u\right) d \mu(t)}{\int_{A} \phi_{1}\left(u_{0}, t\right) d \mu(t)} \\
& -\alpha \frac{\int_{B}\left(\nabla_{w} \phi_{2}\left(w_{0}, x\right), w\right) d \nu(x)}{\int_{B} \phi_{2}\left(w_{0}, x\right) d \nu(x)} .
\end{aligned}
$$

By Theorem 7.5 of $[5],(w, u)$ lies in the cone of $K_{0}$ directions of decrease iff $G^{\prime}\left(w_{0}, u_{0}\right)(w, u)<0$ or, from (3.6), iff

$$
\int_{A}\left(\nabla_{u} \phi_{1}\left(u_{0}, t\right), u\right) d \mu(t)-\alpha \lambda \int_{B}\left(\nabla_{w} \phi_{2}\left(w_{0}, x\right), w\right) d \nu(x)<0,
$$


where $\lambda$ is given by (3.4). If $K_{0} \neq \phi$, then by Theorem 10.5 of [5], for any $g_{0} \in K_{0}^{*}$,

$$
\begin{aligned}
g_{0}(w, u)=-\lambda_{0}\left\{\int_{A}\left(\nabla_{u} \phi_{1}\left(u_{0}, t\right), u\right) d \mu(t)\right. & \\
& \left.-\alpha \lambda \int_{B}\left(\nabla_{w} \phi_{2}\left(w_{0}, x\right), w\right) d \nu(x)\right\}, \quad \lambda_{0} \geq 0 .
\end{aligned}
$$

B. Cone of tangent directions. To find the tangent directions at $\left(w_{0}, u_{0}\right)$ in $E$, we apply the results of Lecture 9 of [5]. Let

$$
Q=\{(w, u) \in E \mid w=D u\}
$$

and

$$
P(w, u)=w-D u,
$$

which maps $E$ into $L_{r}^{n}(B, \nu)$. Also

$$
P^{\prime}\left(w_{0}, u_{0}\right)(w, u)=w-D u .
$$

Clearly $P^{\prime}\left(w_{0}, u_{0}\right)$ is onto $L_{r}^{n}(B, \nu)$ (just let $\left.u=0\right)$. By Theorem 9.1 of [5], the set $K_{1}$ of tangent directions in $E$ at $\left(w_{0}, u_{0}\right)$ is given by

$$
K_{1}=\left\{(w, u) \in E \mid P^{\prime}\left(w_{0}, u_{0}\right)(w, u)=0\right\}=\{(w, u) \in E \mid w=D u\} .
$$

Thus $K_{1}$ is a subspace and, if $g_{1} \in K_{1}^{*}$, then $g_{1}(w, u)=0$ for all $(w, u) \in K_{1}$.

C. Application of the Dubovitskii-Milyutin theorem. Let $u$ be arbitrary and $w=$ $D u$. Then for these pairs of $(w, u)$ we get (since $g_{1}(w, u)+g_{2}(w, u)=0,(w, u) \in$ $\left.E,\left(g_{1}, g_{2}\right) \not \equiv 0\right)$

$$
\int_{A}\left(\nabla_{u} \phi_{1}\left(u_{0}, t\right), u\right) d \mu(t)-\alpha \lambda \int_{B}\left(\nabla_{w} \phi_{2}\left(w_{0}, x\right), w\right) d \nu(x)=0
$$

for all $u$. So

$$
\nabla_{u} \phi_{1}\left(u_{0}, t\right)-\alpha \lambda D^{*}\left(\nabla_{w} \phi_{2}\left(w_{0}, x\right)\right)=0 \text { a.e. }[\mu] \text { on } A \text {. }
$$

D. Exceptional case. If $K_{0}=\emptyset$ we have

$$
\int_{A}\left(\nabla_{u} \phi_{1}\left(u_{0}, t\right), u\right) d \mu(t)-\alpha \lambda \int_{B}\left(\nabla_{w} \phi_{2}\left(w_{0}, x\right), w\right) d \nu(x)=0
$$

for all $(w, u) \in E$. Thus (3.3) is satisfied in this case also.

Corollary 3.1. Let $A=B, \mu=\nu$ and

$$
F(w, u)=\frac{\int_{A} \phi_{1}(w, u, t) d \mu(t)}{\left[\int_{A} \phi_{2}(w, t) d \mu(t)\right]^{\alpha}} .
$$

In addition to assumptions (i)-(vi) of this section assume the following.

(a) $\phi_{1}$ is continuously differentiable in $(w, u)$ and measurable in $t$. Also $\nabla_{w} \phi_{1} \in$ $L_{s}^{n}(A, \mu)$ whenever $w \in L_{r}^{n}(A, \mu), 1 / r+1 / s=1$.

(b) Replace the first inequality in (3.2) by

$$
\begin{aligned}
& \left\|\nabla_{u} \phi_{1}(w+h, u+\bar{u}, t)-\nabla_{u} \phi_{1}(w, u, t)\right\|_{q} \\
& \quad+\left\|\nabla_{w} \phi_{1}(w+h, u+\bar{u}, t)-\nabla_{w} \phi_{1}(w, u, t)\right\|_{s} \\
& \leq k_{1}\left[\|h\|_{r}+\|\bar{u}\|_{p}\right] .
\end{aligned}
$$


If $\left(w_{0}, u_{0}\right)$ minimizes (3.15), then

$$
\nabla_{u} \phi_{1}\left(w_{0}, u_{0}, t\right)+D^{*}\left(\nabla_{w} \phi_{1}\left(w_{0}, u_{0}, t\right)-\alpha \lambda \nabla_{w} \phi_{2}\left(w_{0}, t\right)\right)=0
$$

a.e. $[\mu]$ on $A$, where

$$
\lambda=\frac{\int_{A} \phi_{1}\left(w_{0}, u_{0}, t\right) d \mu(t)}{\int_{A} \phi_{2}\left(w_{0}, t\right) d \mu(t)} .
$$

\section{REFERENCES}

1. M. B. Subrahmanyam, On applications of control theory to integral inequalities, J. Math. Anal. Appl. 77 (1980), 47-59.

2. $\ldots$, On applications of control theory to integral inequalities. II, SIAM J. Control Optim. 19 (1981), 479-489.

3., A control problem with application to integral inequalities, J. Math. Anal. Appl. 81 (1981), 346-355.

4. __ An extremal problem for convolution inequalities, J. Math. Anal. Appl. 87 (1982), 509-516.

5. I. V. Girsanov, Lecture Notes in Econom. and Math. Systems, no. 67, Springer-Verlag, New York, 1972.

Department of Electrical Engineering, University of Missouri-Columbia, COLUMBIA, MisSOURI 65211 